\title{
Hemangioma originating from the main pulmonary artery
}

\author{
In-Sub Kim ${ }^{1}$, Jae Jun Kim ${ }^{1}$, Si Young Choi ${ }^{1}$, Seong Cheol Jeong ${ }^{1}$, Eundeok Chang ${ }^{2}$, Yong Hwan Kim ${ }^{1}$ \\ ${ }^{1}$ Department of Thoracic and Cardiovascular Surgery, ${ }^{2}$ Department of Hospital Pathology, Uijeongbu St. Mary's Hospital, The Catholic University \\ of Korea, College of Medicine, Uijeongbu, Korea \\ Correspondence to: Professor Yong Hwan Kim, MD, PhD. Department of Thoracic and Cardiovascular Surgery, Uijeongbu St. Mary's Hospital, The Catholic \\ University of Korea, College of Medicine, Uijeongbu, Korea, 271, Cheonbo-ro, Uijeongbu-si, Gyeonggi-do, 11765 Korea. Email: cmccs@naver.com.
}

\begin{abstract}
In this study, we report the case of a 42 -year-old female patient who was diagnosed with hemangioma in the pericardium connected to the main pulmonary artery. Chest CT revealed a hypodense, well-marginated, mass-like lesion, probably connected to the main pulmonary artery, and multiple heterogeneous attenuations were seen with contrast enhancement. The tumor was resected using conventional extracorporeal circulation for complete resection involving the main pulmonary artery wall. Pathologic examination revealed hemangioma. Because main pulmonary originated hemangioma is extremely rare, examination of these cases provides much needed data to further our understanding of this disease. We herein report such a case.
\end{abstract}

Keywords: Hemangioma; main pulmonary artery; intrapericardial mass

Submitted Jun 13, 2017. Accepted for publication Aug 21, 2017.

doi: $10.21037 /$ jtd.2017.08.129

View this article at: http://dx.doi.org/10.21037/jtd.2017.08.129

\section{Introduction}

Hemangioma is a rare disease that occurs in about $0.5 \%$ of mediastinal tumors (1). In addition, hemangioma of the main pulmonary artery has been rarely reported. Here, we report the case of a 42 -year-old female patient with incidentally diagnosed hemangioma in the pericardium connected to the main pulmonary artery.

\section{Case presentation}

A 42-year-old female was referred to our hospital because of abnormal chest $\mathrm{X}$-ray during a routine health screening examination. The patient had no specific symptoms, no cardiac murmur, and normal sinus rhythm on the electrocardiogram. Chest X-ray (Figure 1) showed an enlarged, slightly protruding left cardiac border. There were no valve abnormalities and no shunt flow on echocardiography. Chest computed tomography (CT) revealed a hypodense, well-marginated large, mass-like lesion, probably connected to the main pulmonary artery, and confirmed the presence of multiple heterogeneous attenuations with contrast enhancement (Figure 2).
The right ventricular outflow tract was compressed and dislocated to posteriorly by the tumor. Pulmonary angiography and coronary angiography were performed to differentiate pulmonary pseudoaneurysm and to identify the supplying vessel. Coronary angiography showed two supplying vessels from the right coronary artery (RCA) and the left anterior descending artery (LAD) (Figure $3 A, B$ ). There was no evidence of pseudoaneurysm on pulmonary artery angiography (Figure 3C,D). Since the mass involved the pulmonary artery on CT, a median sternotomy was performed for conventional extracorporeal circulation. The mass was about $8 \mathrm{~cm} \times 6 \mathrm{~cm}$ in size and was a wellcircumscribed, multi-lobulated, soft mass without pericardial adhesion in the pericardial space (Figure 4). The main pulmonary artery was resected in the shape of an oval, including the connecting site under conventional extracorporeal circulation, for complete resection. The pulmonary valve was intact in appearance, and the resected pulmonary artery was closed with a bovine pericardial patch (Figure 4). Pulmonary valve function was well maintained as assessed by transesophageal echocardiography after extracorporeal circulation weaning.

On cross section, the tumor showed a blood-filled sponge- 
like septated mass (Figure $5 A$ ). H\&E staining findings were large dilated cystic vessels with intravascular thrombosis, suggesting hemangioma (Figure $5 B$ ). The immunostaining of hemangioma were positive for CD34 (Figure 5C) and

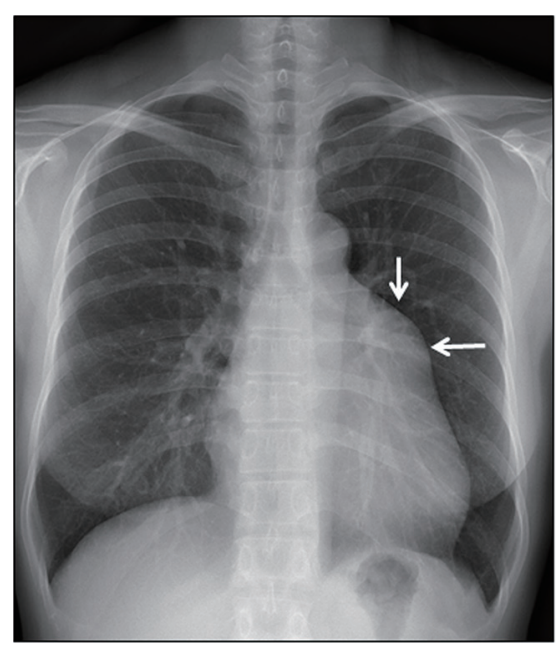

Figure 1 Chest PA: enlarged, protruding left cardiac border (arrow). negative for $\mathrm{p} 53$ in endothelial cells. The proliferative index Ki-67 (MIB-1) was very low, 1\% (Figure 5D, × 200).

The patient was discharged without problem. Three months after the operation, the follow-up chest CT showed no residual mediastinal mass.

\section{Discussion}

Primary cardiac tumors can be divided into intracavitary, mural, and intrapericardial types, depending on location (2). Intrapericardial tumors can be further divided into primary cardiac and noncardiac, according to origin (3). Primary intrapericardial tumors are very rare, are almost benign, and usually originate from the myocardium (2). There have been several reports of hemangioma in the epicardium (4-6). However, hemangioma on the main pulmonary artery has been rarely been reported. In 1959, Umehara reported hemangioma on the surface of the pulmonary artery, but no literature or images of that case could be obtained (7). Although a giant hemangioma of the anterior mediastinum with left pulmonary artery adhesion has been reported, the tumor did not originate from the pulmonary
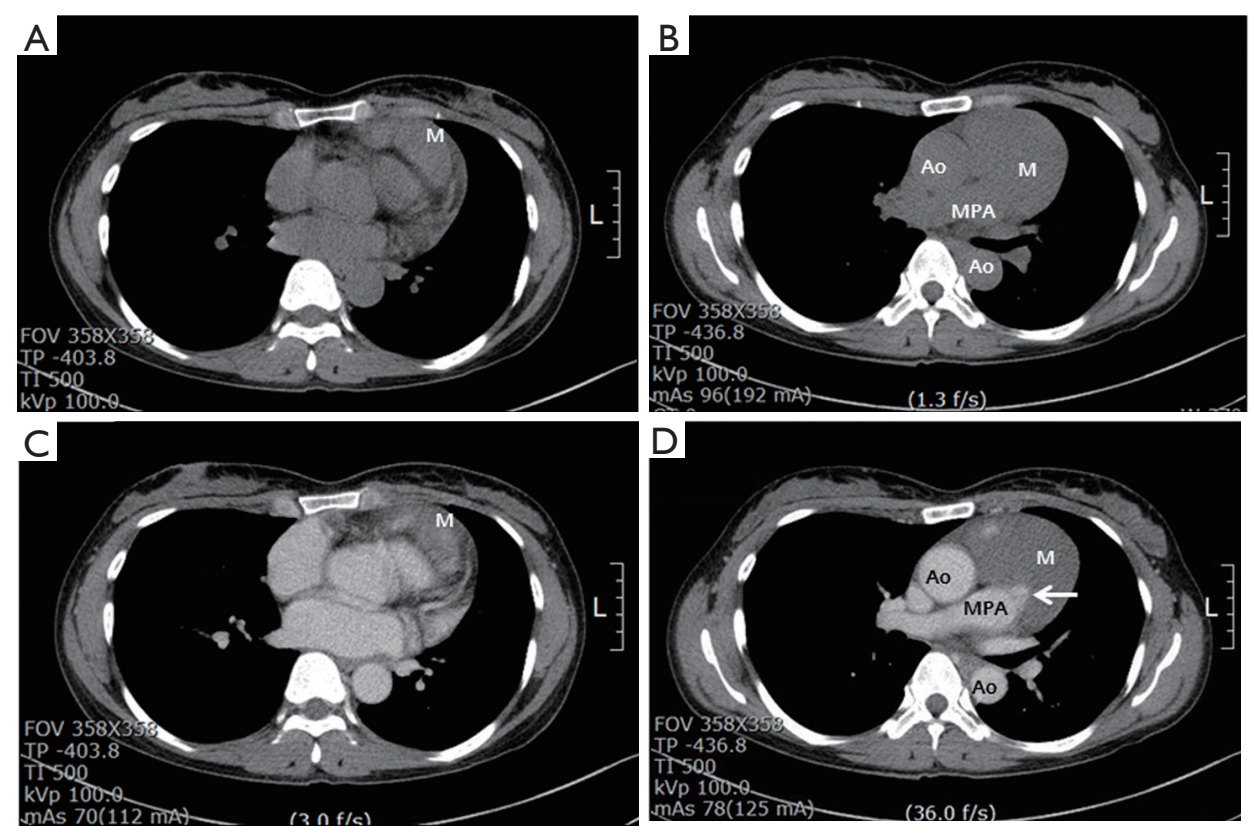

Figure 2 Chest CT revealed a hypodense well-marginated mass-like lesion, probably connected to the main pulmonary artery, and confirmed the presence of multiple heterogeneous attenuations with contrast enhancement. (A,B) Pre-enhanced CT; (C,D) multiple heterogeneous attenuations with contrast enhancement; the right ventricular outflow tract was compressed and dislocated to posteriorly by the tumor; (D) the tumor is probably connected to the pulmonary artery (arrow). CT, computed tomography; Ao, aorta; MPA, main pulmonary artery; $M$, mass. 

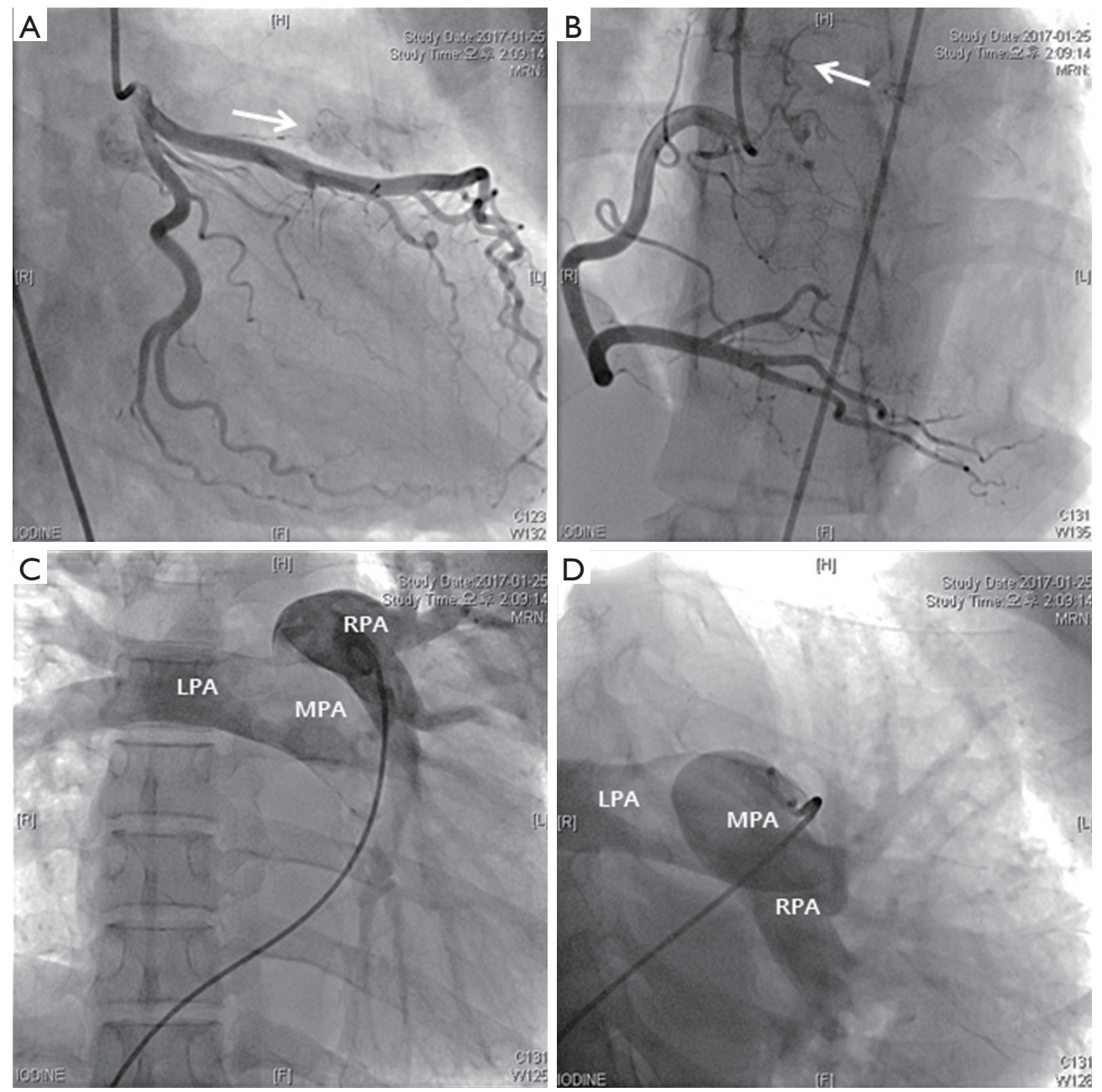

Figure 3 Coronary and pulmonary angiography. (A) Supplying vessel from the left anterior descending artery (arrow); (B) supplying vessel from the proximal right coronary artery (RCA) (arrow); (C,D) angiography of the main pulmonary artery showed no aneurysmal change. LPA, left pulmonary artery; MPA, main pulmonary artery; RPA, right pulmonary artery.

artery (8). In this case, we suspected a benign mediastinal tumor or benign cardiac tumor because the border of the tumor was relatively clear. However, CT showed that the tumor and the main pulmonary lumen were connected, and pseudoaneurysm could not be excluded. Therefore, pulmonary angiography was performed, but no pseudoaneurysm was found. Pericardial tumors are known to originate from arteries that supply the coronary arteries (9). We performed coronary angiography because accurate identification of the origin of the blood supply is necessary for proper surgical resection of the pericardial tumor $(3,9)$ and discovered the origin to be two supplying arteries connected to the RCA and LAD.

Most cases of pericardial tumors are known to be asymptomatic, but involvement of the pericardium can cause chest pain, hemopericardium, and cardiac tamponade $(10,11)$. In this case, the patient did not have any symptoms, and there was no involvement of the pericardium in the surgical findings. The lack of symptoms is likely attributed to the tumor not invading the pericardium and the absence of compression of the pulmonary artery and the heart.

Primary pericardial tumors are mostly benign, but complete resection is necessary because of the possibility of recurrence (3). In our case, because the tumor was connected to the main pulmonary artery, we performed tumor resection, including pulmonary resection, under extracorporeal circulation support.

A well-circumscribed mediastinal tumor with 

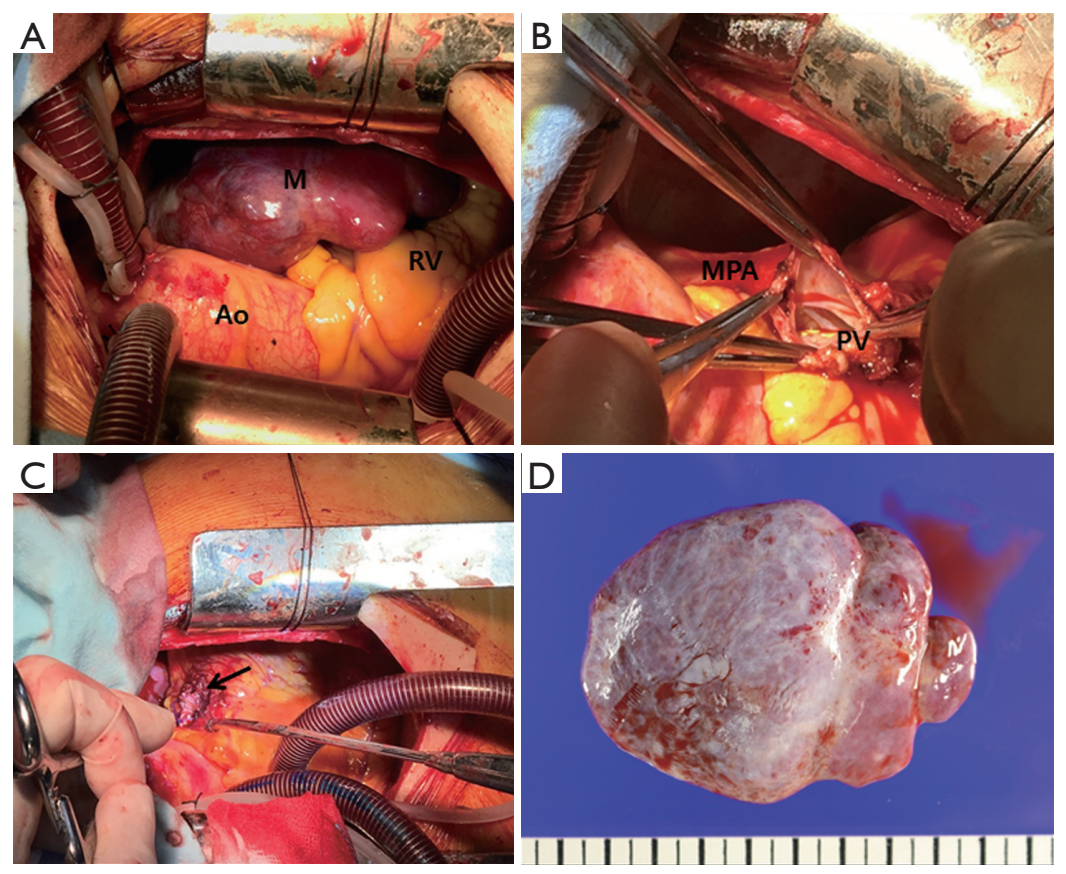

Figure 4 Operative findings. (A) A large mass was found in the intrapericardial space. There was no adhesion to the pericardium or other cardiac surface, except the main pulmonary artery; (B) the pulmonary valve was intact after resection of the tumor involving the main pulmonary artery; (C) the main pulmonary artery was closed with a bovine pericardial patch (arrow); (D) the resected tumor was a multilobulated soft mass. Ao, aorta; MPA, main pulmonary artery; RV, right ventricle; PV, pulmonary valve; M, mass.
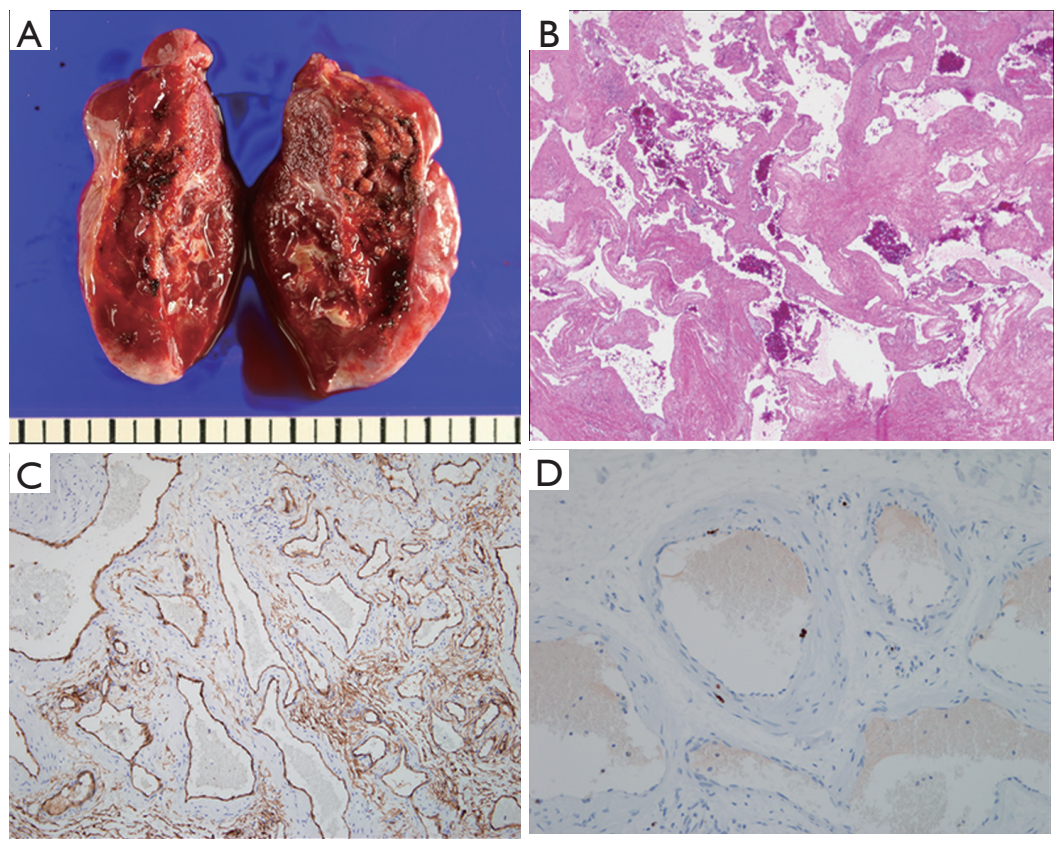

Figure 5 Resected tumor. (A) The cross-section of the tumor was a diffuse, dense, blood-filled, soft, sponge-like mass that was partly septated; (B) H\&E stain findings were large cystically dilated vessels with intravascular thrombosis $(\times 100)$; (C) the immunostaining of hemangioma were positive for CD34 (× 100); (D) the proliferative index Ki-67 (MIB-1) was very low, 1\% $(\times 200)$. 
heterogeneous attenuation and phleboliths are diagnostic features of hemangioma that are revealed on CT scan $(12,13)$. Although this case seemed to fit the appearance of hemangioma, except for the lack of phleboliths, hemangioma was not included in the initial diagnosis because it is so rare. Given this case, hemangioma located in the mediastinum, although its prevalence is low, should be included in the differential diagnosis if the patient presents with characteristic radiological findings.

Here, we report a case of hemangioma originating from the main pulmonary artery, which has not been reported previously.

\section{Acknowledgements}

None.

\section{Footnote}

Conflicts of Interest: The authors have no conflicts of interest to declare.

Informed Consent: Written informed consent was obtained from the patient for publication of this case report and any accompanying images.

\section{References}

1. Cohen AJ, Sbaschnig RJ, Hochholzer L, et al. Mediastinal hemangiomas. Ann Thorac Surg 1987;43:656-9.

2. Goldberg HP, Steinberg I. Primary tumors of the heart.

Cite this article as: Kim IS, Kim JJ, Choi SY, Jeong SC, Chang E, Kim YH. Hemangioma originating from the main pulmonary artery. J Thorac Dis 2017;9(10):E894-E898. doi: 10.21037/jtd.2017.08.129
Circulation 1955;11:963-70.

3. Taguchi S, Yozu R. Surgery for primary intrapericardial tumors in adults. J Card Surg 2013;28:529-32.

4. Hara T, Ushijima T, Takemura H, et al. A case report of cavernous hemangioma of the left atrial epicardium. Kyobu Geka 1998;51:1027-9.

5. Tomizawa Y, Endo M, Nishida H, et al. Reconstruction of the left ventricle in a patient with cardiac hemangioma at the apex. Ann Thorac Surg 2001;71:2032-4.

6. Shikata D, Nakagomi T, Yokoyama Y, et al. Debulking surgery for venous hemangioma arising from the epicardium: report of a case. World J Surg Oncol 2017;15:81.

7. Umehara Y. Hemangiomas of the heart and pulmonary artery surface--a case report. Acta Pathol Jpn 1959;9:821-2.

8. Kaya SO, Samancilar O, Usluer O, et al. Giant Cavernous Haemangioma of the Anterior Mediastinum. Eurasian J Med 2015;47:216-7.

9. Chang CH, Lin PJ, Chang JP, et al. Intrapericardial pheochromocytoma. Ann Thorac Surg 1991;51:661-3.

10. Kojima S, Sumiyoshi M, Suwa S, et al. Cardiac hemangioma: a report of two cases and review of the literature. Heart Vessels 2003;18:153-6.

11. Butany J, Nair V, Naseemuddin A, et al. Cardiac tumours: diagnosis and management. Lancet Oncol 2005;6:219-28.

12. McAdams HP, Rosado-de-Christenson ML, Moran CA. Mediastinal hemangioma: radiographic and CT features in 14 patients. Radiology 1994;193:399-402.

13. Agarwal PP, Seely JM, Matzinger FR. Case 130: Mediastinal Hemangioma. Radiology 2008;246:634-7. 